\title{
AVALIAÇÃO CLÍNICA, RADIOGRáFICA E LABORATORIAL DE PACIENTES COM OSTEOGÊNESE IMPERFEITA
}

\author{
Cláudio Santili*, Miguel Akkari, Gilberto Waisberg, José Olympio Catäo Bastos Júnior, William Martins ferreira \\ Trabalho realizado no Grupo de Ortopedia Pediátrica do Departamento de Ortopedia e Traumatologia do Hospital Central da Faculdade de \\ Ciências Médicas da Santa Casa de São Paulo, São Paulo, SP.
}

\author{
* Correspondência: \\ Departamento de Ortopedia \\ e Traumatologia da \\ Irmandade da Santa Casa \\ de Misericórdia de São \\ Paulo. Grupo de Ortopedia \\ Pediátrica do Hospital e da \\ Faculdade de Ciências \\ Médicas da Santa Casa de \\ São Paulo - Rua Cesário \\ Motta Jr. 112, Vila Buarque, \\ São Paulo, SP, Cep 01277. \\ 900, Fone (11) 221-2395 \\ c.santili@terra.com.br
}

\begin{abstract}
RESUMO
OBjETIVos. A osteogênese imperfeita $(\mathrm{Ol})$ é uma doença genética, caracterizando-se por alterações no colágeno do tipo I, que determinam um espectro amplo de alterações clínicas, como a dentinogênese imperfeita e escleras azuladas. O objetivo deste estudo é estabelecer uma correlação prática no diagnóstico diferencial intergrupos dentro da classificação de Sillence et al. (1979).

Métooos. Foram avaliados 22 pacientes mediante critérios clínicos e radiográficos. Após, a subdivisão de acordo com os tipos de Sillence et al. (1979), os pacientes foram também submetidos à avaliação laboratorial e à densitometria óssea.

Resultados. Os dados significantes para diferenciaçãao entre os tipos da doença foram a estatura, o número total de fraturas por indivíduo e a densitometria óssea. O cálcio sérico não diferencia os tipos da doença.

Concusós.s. Características como a deambulação, a estatura e a densitometria óssea podem auxiliar na diferenciação entre os subtipos dos portadores da doença, repercutindo diretamente no estabelecimento do seu prognóstico.
\end{abstract}

UNITERMOS: Osteogênese imperfeita. Escleras azuis. Dentinogênese imperfeita.

\section{INTRODUÇÃo}

A osteogênese imperfeita é uma doença determinada geneticamente, na qual está afetada a estrutura e a função do colágeno do tipo I, que representa mais de $90 \%$ do colágeno tecidual total, sendo responsável por $70 \%$ a $80 \%$ do peso seco dos tecidos fibrosos densos que formam o sistema músculo-esquelético'. O padrão de herança mais comum é o autossômico dominante, podendo ser, com menor freqüência, recessivo. A alteração é determinada por mutações nos cromossomos 7 e 17, mais especificamente nos Loci COLIAI e COLIA2, onde ocorre com maior freqüência a substituição da glicina por um outro aminoácido; mutações localizadas nestes Loci determinam principalmente a Ol, mas podem também causar, dentre outras doenças, a síndrome de Ehlers-Danlos dos tipos VIIA ou VIIB ${ }^{2,3}$. Um grande número de mutações tem sido descrito, porém a correlação entre o defeito genético específico e o fenótipo produzido ainda é pouco estabelecida ${ }^{2}$.

A Ol é uma doença rara, ocorrendo um caso em cada 15.000 a 20.000 nascimentos e sua prevalência é de I em 200.000 indivíduos ${ }^{4}$. Não há citações na literatura sobre o predomínio em relação à raça ou sexo.

A apresentação clínica é heterogênea e variável sendo, no entanto, marcantes a fragilidade óssea, a frouxidão cápsulo-ligamentar, a cor azulada da esclera e a surdez ${ }^{5,6,7}$. No quadro laboratorial, a fosfatase alcalina sérica acha-se freqüentemente aumentada, não sendo observadas, porém, alterações nos níveis sistêmicos do cálcio, fósforo, hormônio da paratireóide ou mesmo da I,25 vitamina $\mathrm{D}^{1,6,6,9,10,11}$. Estudos mais recentes mostram aumento na excreção urinária de cálcio, fósforo, magnésio, hidroxiprolina e glicosaminoglicanos ${ }^{7}, 12,13$. Pode ser encontrada aminoacidúria, com a conseqüente diminuição dos aminoácidos séricos ${ }^{6,89}$ e, mais raramente, podem ocorrer alterações metabólicas que elevam o risco de sangramentos e maior predisposição à hipertermia maligna $a^{8,9}$.

O diagnóstico habitualmente é feito mediante a história clínica, o aspecto ao exame físico e constatações radiográicas, não existindo exame complementar de uso prático que seja espećíico para a confirmação da doença, nem tampouco uma adequada correlação clínico-laboratorial para cada tipo de apresentação fenotípica da classificação de Sillence et al ${ }^{4}$.

No presente estudo, são apresentados os resultados obtidos na avaliação clínica e laboratorial de 22 pacientes portadores da doença, investigados com o objetivo de estabelecer uma correlação prática no diagnóstico diferencial entre os tipos clínicos.

\section{Métodos}

Foram avaliados clínica e laboratorialmente 22 pacientes, no período de dezembro de 1999 a junho de 2000, no Departamento de Ortopedia e Traumatologia da Santa Casa de Misericórdia de São Paulo. O grupo foi constituído por 14 pacientes do sexo feminino $(63,6 \%)$ e oito do sexo masculino (36,4\%), e a média de idade foi de 22,2 anos (variando de 2 a 54 anos). Destes, apenas nove (40,9\%) apresentavam antecedentes familiares da doença.

Quanto à distribuição por raça, $13(59,1 \%)$ eram brancos, cinco $(22,7 \%)$ pardos e quatro $(18,2 \%)$ negros.

Considerando que estes pacientes psicologicamente estressam-se muito no ambiente hospitalar, criamos um período especial para o seu atendimento ambulatorial isolado.

Todos os pacientes ou seus responsáveis receberam o consentimento informado e concordaram com seus termos. 
O protocolo de avaliação clínica constava de identificação completa do paciente, e incluía dados quanto ao peso e estatura, presença ou não de dentinogênese alterada, coloração das escleras, antecedentes familiares da doença, idade na primeira fratura, número total de fraturas, interrogatório sobre a capacidade auditiva e a evolução da marcha até o estado atual de capacitação deambulatória, além da relação das deformidades músculo-esqueléticas no momento do estudo.

A avaliação da estatura com diferencial quanto ao sexo e idade foi cotejada com os dados normais da tabela e desvios-padrão da população brasileira $^{14}$.

A avaliação radiográfica foi baseada em radiografias nas incidências ântero-posterior e perfil dos ossos já fraturados. Incluiu também a coluna vertebral nos casos de deformidades constatadas no exame clínico, além da radiografia da bacia abrangendo o fêmur proximal em todos os casos. As radiografias foram analisadas quanto à presença de deformidades, espessura da cortical femoral e observação macroscópica da densidade do trabeculado ósseo na medular no fêmur proximal. A análise radiográfica foi realizada por três ortopedistas e um radiologista, prevalecendo em todos os casos o consenso da maioria. Quanto aos critérios de classificação para a escoliose, consideramos que valores angulares menores que $20^{\circ}$ eram leves, valores entre $20^{\circ}$ e $40^{\circ}$ moderados e acima de $40^{\circ}$ eram graves.

Após a avaliação clínica e radiográfica, os pacientes foram classificados segundo os critérios da classificaçãa de Sillence et al. ${ }^{4}$ (Figura I).

$\mathrm{Na}$ avaliação laboratorial, foram realizados a dosagem sérica de cálcio e o exame de densitometria óssea de corpo total em todos os pacientes. Foi utilizado o método da densitometria duo-energética (DEXA), e em todos os casos foi utilizado o mesmo aparelho (LUNAR), na Unidade de Densitometria Óssea do Hospital. Com este método se realiza a mensuração da densidade mineral óssea do esqueleto e, após a correção de fatores raciais e antropométricos, os resultados são comparados com um padrão de normalidade para a mesma idade, peso, sexo e raça do paciente. Os resultados são finalmente expressos em desvios-padrão (DP) da normalidade.

Não tivemos pacientes classificados como tipo II de Sillence et al. ${ }^{4}$, e por isso foram analisados apenas três diferentes grupos, cotejando-se a estatura (dada em desvios-padrão para a idade e sexo), o número total de fraturas, a dosagem do cálcio e a densitometria óssea (dada em desvios-padrão para a idade, sexo, peso e raça).

Para a análise estatística, aplicamos o teste não paramétrico de Mann-Whitney, utilizando-se o software S.P.S.S. 8. OforWindows.

\section{Resultados}

Quanto à classificação pelos critérios de Sillence et al. ${ }^{4}$ para osteogênese imperfeita, obtivemos nove pacientes portadores do tipo I, cinco pacientes do tipo III e oito do tipo IV.

\section{Grupo de pacientes portadores do Tipo I de Sillence et al. ${ }^{4}$}

Dos nove pacientes classificados como portadores do tipo I, oito eram mulheres (88,9\%) e um homem ( I I, I\%), com uma média de idade de 22 anos (variando de 4 a $4 \mathrm{I}$ anos). Cinco deles (55,5\%) tinham história familiar da doença. A média de idade em que ocorreu a primeira fratura foi aos 2,7 anos, variando desde o nascimento até os nove anos de idade. Ocorreram no total 70 fraturas neste grupo, gerando uma média de 7,8 fraturas por paciente. Naquele momento, todos estes pacientes eram deambuladores e apresentavam as escleras azuis.

A diminuição da capacidade auditiva confirmada por avaliação otorrinolaringológica foi constatada em dois pacientes (22,2\%). Seis pacientes $(66,7 \%)$ eram do subtipo IA de Sillence et al. ${ }^{4}$, pois apresentavam dentinogênese normal $(A)$ e três $(33,3 \%)$ eram do subtipo IB, pois a dentinogênese estava alterada $(B)$. Em relação à estatura, obtivemos que neste grupo a média estava 2,2 desviospadrão (DP) abaixo da normalidade, sendo que cinco pacientes (55,5\%) tinham a estatura abaixo do percentil 2,5. A dosagem de cálcio esteve abaixo do valor de referência em seis pacientes $(66,7 \%)$ (Tabela I e Figuras I, 2 e 3).

Com relação à avaliaçãa radiográfica, encontramos alterações com diminuição na espessura da cortical e/ou diminuição do trabeculado ósseo da medular no fêmur proximal em três pacientes. Havia comprovação de escoliose moderada sem alterações neurológicas em dois pacientes (22\%). (Tabela I).

$\mathrm{Na}$ avaliação da massa óssea pela densitometria, apenas num paciente $\left(\mathrm{I} \mid \mathrm{I}\right.$ \%), dos nove classificados como tipo I de Sillence et al. ${ }^{4}$, o resultado foi inferior ao esperado, estando 2,8 DP abaixo da normalidade, correspondendo à média de 0,3। DP para os pacientes do grupo I (Tabela I e Figura 3).

\section{Grupo de pacientes portadores do Tipo III de Sillence et al. ${ }^{4}$}

Neste grupo, todas as cinco pacientes eram do sexo feminino com média de idade de 27,6 anos (variando entre 10 e 54 anos). Três pacientes (60\%) tinham história familiar da doença. O primeiro episódio da fratura ocorreu no nascimento em quatro (80\%) destas pacientes e com apenas um mês de vida na outra. No total, ocorreram 202 fraturas até aquele momento gerando uma média de 40,4 fraturas por paciente. Nenhuma delas chegou a deambular em algum momento de suas vidas, e todas apresentavam dentinogênese imperfeita. A audição era normal em todas elas e quatro (80\%) apresentavam escleras azuis. Neste grupo a média de DP em relação a estatura foi 7,5I DP abaixo da normalidade e todas tinham a estatura abaixo do percentil 2,5. A dosagem de cálcio esteve abaixo do valor de referência em todas estas pacientes (Tabela 2 e Figuras I, 2 e 3).

$\mathrm{Na}$ avaliação radiográfica, observamos que todas apresentavam diminuição na espessura da cortical e do trabeculado ósseo da medular no fêmur proximal; além disso, havia escoliose grave em quatro (80\%) e moderada em uma paciente (Tabela 2).

$\mathrm{Na}$ avaliação da massa óssea pela densitometria, em todas as pacientes deste grupo obtivemos resultados abaixo do esperado, sendo a média 3,4 DP abaixo da normalidade (Tabela 2 e Figura 3).

\section{Grupo de pacientes portadores do Tipo IV de Sillence et al. ${ }^{4}$}

Foram classificados como portadores do tipo IV da doença oito pacientes (36,4\% do total); sendo uma mulher (12,5\%) e sete homens (87,5\%), com uma média de idade de 19,I anos (variando de 2 a 41 ). Apenas um paciente $(12,5 \%)$ deste grupo tinha história familiar da doença. O primeiro episódio de fratura ocorreu com uma média de 9,2 meses de vida (variando desde o nascimento até os três anos de idade). No total ocorreram I 55 fraturas neste grupo, gerando uma média de 


\begin{tabular}{|c|c|c|c|c|c|c|c|c|c|}
\hline Iniciais & ERD & APSB. & TPO & $\mathrm{COA}$ & POA & FGAC & CMB & MMS & OOS \\
\hline Sexo & $F$ & $\mathrm{~F}$ & $F$ & $F$ & $F$ & M & $\mathrm{F}$ & $\mathrm{F}$ & $F$ \\
\hline Idade (anos) & 41 & $\|$ & 39 & 13 & 19 & 4 & $\|$ & 21 & 39 \\
\hline Estatura $(\mathrm{cm})$ & 138 & 154 & 133 & 135 & 152 & 105 & 125 & $|5|$ & 135 \\
\hline Antecedentes familiares & Não & Não & Sim & Sim & Sim & Não & Não & Sim & Sim \\
\hline Idade $I^{a}$ fratura (meses) & 24 & 18 & 108 & 24 & 72 & 6 & 36 & 0 & 0 \\
\hline Número total de fraturas & 20 & 10 & 4 & 4 & 2 & 5 & 6 & 4 & 15 \\
\hline Escleras azuis & Sim & Sim & Sim & Sim & Sim & Sim & Sim & Sim & Sim \\
\hline Dentinogênese imperfeita & Não & Não & Não & Não & Não & Sim & Sim & Não & Sim \\
\hline Classificação & IA & IA & IA & IA & $\mathrm{A}$ & $\mid B$ & IB & $\mid A$ & IB \\
\hline Audição & $\mathrm{nl}$ & $\mathrm{nl}$ & $\downarrow$ & $\mathrm{nl}$ & $\mathrm{nl}$ & $\mathrm{nl}$ & $\mathrm{nl}$ & $\mathrm{nl}$ & $\downarrow$ \\
\hline Estado deambulatório & $\begin{array}{c}\text { sem auxilio } \\
\downarrow E C\end{array}$ & $\begin{array}{c}\text { sem auxilio } \\
\text { EC nl }\end{array}$ & $\begin{array}{c}\text { sem auxilio } \\
\text { EC } n l\end{array}$ & $\begin{array}{c}\text { sem auxilio } \\
\text { EC nl }\end{array}$ & $\begin{array}{c}\text { sem auxilio } \\
\text { EC } n l\end{array}$ & $\begin{array}{c}\text { sem auxilio } \\
\text { EC } n l\end{array}$ & $\begin{array}{c}\text { sem auxilio } \\
\text { EC nl }\end{array}$ & $\begin{array}{c}\text { sem auxilio } \\
\text { EC nl }\end{array}$ & $\begin{array}{c}\text { sem auxilio } \\
\downarrow \in E C\end{array}$ \\
\hline Avaliação radiográfica & $\begin{array}{l}\downarrow T O \\
\text { sem E }\end{array}$ & $\begin{array}{l}\text { TO nl } \\
\text { sem E }\end{array}$ & $\begin{array}{c}\text { TO nl } \\
\text { EM }\end{array}$ & $\begin{array}{l}\text { TO nl } \\
\text { EM }\end{array}$ & $\begin{array}{l}\text { TO nl } \\
\text { sem E }\end{array}$ & $\begin{array}{l}\text { TO nl } \\
\text { sem E }\end{array}$ & $\begin{array}{l}\downarrow T O \\
\text { sem E }\end{array}$ & $\begin{array}{l}\text { TO nl } \\
\text { sem E }\end{array}$ & $\begin{array}{l}\text { TO nl } \\
\text { sem E }\end{array}$ \\
\hline Densitometria óssea & $2.8 \mathrm{DP}$ & $\mathrm{nl}$ & $\mathrm{nl}$ & $\mathrm{nl}$ & $\mathrm{nl}$ & $\mathrm{nl}$ & $\mathrm{nl}$ & $\mathrm{nl}$ & $\mathrm{nl}$ \\
\hline Cálcio sérico (mg\%) & 9.4 & 9.7 & 9.9 & 9.9 & 9.6 & 8.5 & 8.5 & 9.0 & 10.1 \\
\hline
\end{tabular}

Fonte: Serviço de arquivo médico e estatístico da Irmandade da Santa Casa de Misericórdia de São Paulo.

$\mathrm{nl}=$ normal; $\mathrm{EC}=$ espessura da cortical no fêmur proximal; $\mathrm{TO}=$ trabeculado ósseo da medular no fêmur proximal; $\mathrm{A}=$ dentinogênese normal; $\mathrm{B}=$ dentinogênese alterada; valor de referência do cálcio= 9.9mg\%; $\mathrm{E}=$ escoliose; $\mathrm{EM}=$ escoliose moderada; $\downarrow=$ diminuição.

\begin{tabular}{|c|c|c|c|c|c|}
\hline Iniciais & MAS & $A L N$ & JGE & $10 M$ & M M M \\
\hline Sexo & F & F & $\mathrm{F}$ & F & F \\
\hline Idade (anos) & 54 & 10 & 17 & 15 & 42 \\
\hline Estatura $\quad(\mathrm{cm})$ & 100 & 108 & 100 & 120 & 115 \\
\hline Antecedentes familiares & Sim & $\operatorname{Sim}$ & Não & $\operatorname{Sim}$ & Não \\
\hline Idade Ia fratura (meses) & I & 0 & 0 & 0 & 0 \\
\hline Número total de fraturas & 80 & 15 & 22 & 25 & 60 \\
\hline Escleras azuis & Sim & Sim & Não & Sim & Sim \\
\hline Dentinogênese imperfeita & Sim & Sim & Sim & Sim & $\operatorname{Sim}$ \\
\hline Classificação & |l| & |l| & ||| & || & |l| \\
\hline Audição & $\mathrm{nl}$ & $\mathrm{nl}$ & $\mathrm{nl}$ & $\mathrm{nl}$ & $\mathrm{nl}$ \\
\hline Estado deambulatório & não deambula & não deambula & não deambula & não deambula & não deambula \\
\hline Avaliação radiográfica & $\downarrow E C \downarrow T O$ & $\downarrow E C \downarrow T O$ & $\downarrow E C \downarrow T O$ & $\downarrow E C \downarrow T O$ & $\downarrow E C \downarrow T O$ \\
\hline & EG & EM & EG & EG & EG \\
\hline Densitometria óssea & $4,1 \mathrm{DP}$ & $5,4 \mathrm{DP}$ & 3,0 DP & $2,5 \mathrm{DP}$ & ।,8 DP \\
\hline Cálcio sérico (mg\%) & 9,1 & 8,3 & 9,3 & 9,8 & 8,1 \\
\hline
\end{tabular}

Fonte: Serviço de arquivo médico e estatístico da Irmandade da Santa Casa de Misericórdia de São Paulo.

$\mathrm{nl}=$ normal; $\mathrm{EC}=$ espessura da cortical no fêmur proximal; $\mathrm{TO}=$ trabeculado ósseo da medular no fêmur proximal; valor de referência do cálcio= $9.9 \mathrm{mg} \%$; $\mathrm{E}=$ escoliose; $\mathrm{EM}=$ escoliose moderada; $\mathrm{EG}=$ escoliose grave; $\downarrow=$ diminuição.

19,4 fraturas por paciente. Todos apresentavam audição normal e sete tinham escleras azuis (87,5\%). Três pacientes eram do subtipo IVB (37,5\%), pois apresentavam dentinogênese imperfeita, enquanto que, cinco (62,5\%) não apresentavam esta alteração (subtipo IVA). Quanto à deambulação, três (37,5\%) deambulavam sem auxílio, dois (25\%) deambulavam com muletas e três $(37,5 \%)$ não mais deambulavam. Todos, porém, já haviam deambulado em alguma época da vida. Neste grupo a média de DP em relação à estatura foi de 3,2 DP abaixo da normalidade e cinco pacientes (62,5\%) apresentam estatura abaixo do percentil 2,5. A dosagem de cálcio esteve abaixo do valor de referência em sete (87,5\%) pacientes (Tabela 3 e Figuras I, 2 e 3).

Com relação à avaliação radiográfica, identificamos que em sete destes pacientes (87,5\%) havia diminuição na espessura da cortical e do trabeculado ósseo da medular no fêmur proximal. Constatamos também a presença de escoliose moderada em cinco pacientes e grave em dois. Na avaliação do paciente mais jovem de toda a amostra, com dois anos de idade, não observamos alteração na cortical ou no trabeculado ósseo medular, não havendo também escoliose, apesar 


\begin{tabular}{|c|c|c|c|c|c|c|c|c|}
\hline Iniciais & $R V R$ & AVC & MSPM & LAA & WBS & RB & $J P L$ & WSB \\
\hline Sexo & $\mathrm{F}$ & M & M & M & M & M & M & M \\
\hline Idade (anos) & 41 & 28 & 10 & 20 & 12 & 17 & 2 & 23 \\
\hline Estatura $\quad(\mathrm{cm})$ & 138 & 150 & 150 & 160 & 115 & $\|$ & 80 & 158 \\
\hline Antecedentes familiares & Não & Não & Não & Não & Não & Não & Sim & Não \\
\hline Idade $I^{a}$ fratura (meses) & 36 & 3 & 0 & 9 & 2 & 0 & 0 & 24 \\
\hline Número total de fraturas & 25 & 35 & 9 & 20 & 8 & 38 & 8 & 12 \\
\hline Escleras azuis & Sim & Sim & Sim & Sim & Não & Sim & Sim & Sim \\
\hline Dentinogênese imperfeita & Sim & Sim & Não & Não & Não & Não & Sim & Não \\
\hline Classificação & IV B & IV B & IVA & IVA & IVA & IVA & IV B & IVA \\
\hline Audição & $\mathrm{nl}$ & $\mathrm{nl}$ & $\mathrm{nl}$ & $\mathrm{nl}$ & $\mathrm{nl}$ & $\mathrm{nl}$ & $\mathrm{nl}$ & $\mathrm{nl}$ \\
\hline Estado deambulatório & sem auxílio & uso de muletas & não deambula & não deambula & uso de muletas & não deambula & sem auxílio & sem auxilio \\
\hline Avaliação radiográfica & $\downarrow E C$ & $\downarrow E C$ & $\downarrow E C$ & $\downarrow E C$ & $\downarrow E C$ & $\downarrow E C$ & $\mathrm{EC} \mathrm{nl}$ & $\downarrow E C$ \\
\hline & $\downarrow T O$ & $\downarrow T O$ & $\downarrow T O$ & $\downarrow T O$ & $\downarrow T O$ & $\downarrow T O$ & TO $\mathrm{nl}$ & $\downarrow T O$ \\
\hline & EM & EM & EM & EM & EM & EG & $\operatorname{sem} E$ & $E G$ \\
\hline Densitometria óssea & $4,3 \mathrm{DP}$ & $3,9 \mathrm{DP}$ & $1,78 \mathrm{DP}$ & $4,5 \mathrm{DP}$ & $1,07 \mathrm{DP}$ & $5,26 \mathrm{DP}$ & $\downarrow$ & $3,7 \mathrm{DP}$ \\
\hline Cálcio sérico $(\mathrm{mg} \%)$ & 9,8 & 8,5 & 9,7 & 10,5 & 8,9 & 8,4 & 9,6 & 9,8 \\
\hline
\end{tabular}

Fonte: Serviço de arquivo médico e estatístico da Irmandade da Santa Casa de Misericórdia de São Paulo.

$\mathrm{n}=$ normal; $\mathrm{EC}=$ espessura da cortical no fêmur proximal; $\mathrm{TO}=$ trabeculado ósseo da medular no fêmur proximal; $\mathrm{A}=$ dentinogênese normal; $\mathrm{B}=$ dentinogênese alterada; valor de referência do cálcio=

$9.9 \mathrm{mg} \%$; $E=$ escoliose; $E M=$ escoliose moderada; $E G=$ escoliose grave; $\downarrow=$ diminuição

das graves deformidades nas coxas e pernas e do antecedente de oito fraturas até aquele momento (Tabela 3).

$\mathrm{Na}$ avaliação da massa óssea havia resultados abaixo do esperado em todos os pacientes deste grupo, apresentando a média 3,5 DP abaixo da normalidade. Neste grupo, o resultado da densitometria óssea no paciente de dois anos de idade não foi incluído por não existir padrão de comparação na aplicação desta técnica com este tipo de aparelho para pacientes com esta idade (o resultado foi dado como abaixo do valor esperado, mas sem desvio-padrão) (Tabela 3 e Figura 3).

Os resultados estatísticos quanto às diferentes variáveis na comparação entre os três grupos foram os seguintes:

\section{Estatura média do grupo:}

Resultados do tipo I vs tipo III de Sillence et al. ${ }^{4}: p=0,00$ | (significante).

Resultados do tipo I vs tipo IV de Sillence et al. ${ }^{4}: p=0,48$ (não significante).

Resultados do tipo III vs tipo IV de Sillence et al. ${ }^{4}: p=0,0$ I I (significante).

\section{Número total de fraturas:}

Resultados do tipo I vs tipo III de Sillence et al. ${ }^{4}: p=0,002$ (significante).

Pacientes do tipo I vs tipo IV de Sillence et al. ${ }^{4}: p=0,015$ (significante).

Resultados do tipo III vs tipo IV de Sillence et al. ${ }^{4}: p=0,127$ (não significante).

\section{Dosagem de cálcio}

Todos os valores abaixo da referência, no entanto, sem diferença estatisticamente significante na comparação entre os três diferentes tipos.

\section{Densitometria óssea:}

Resultados do tipo I vs tipo III de Sillence et al. ${ }^{4}: p=0,004$ (significante)

Resultados do tipo I vs tipo IV de Sillence et al. ${ }^{4}: p=0,00$ I (significante).

Resultados do tipo III vs tipo IV de Sillence et al. ${ }^{4}: p=1,00$ (não significante).

\section{Discussão}

A osteogênese imperfeita $(\mathrm{Ol})$ é causada por um defeito quantitativo e qualitativo no colágeno do tipo I ${ }^{(5,8)}$. O colágeno do tipo I é um heteropolímero constituído por duas cadeias alfa I e uma cadeia alfa 2 , que assumem estruturalmente a forma de tripla hélice, e sua determinação genética ocorre nos Loci COLIAI e COLIA2. A herança obedece um padrão autossômico dominante, ou, menos freqüentemente, recessivo. Encontramos antecedentes de história familiar em apenas 40,9\% dos nossos pacientes, mas não pudemos estabelecer o padrão de herança genética que envolve estes doentes.

Embora não haja citação na literatura sobre o tipo de prevalência em relação à raça ou mesmo quanto ao sexo, na amostra analisada houve um leve predomínio do sexo feminino em relação ao masculino, na proporção de I,7 para um, e uma distribuição racial com 59, I \% de brancos, 22,7\% de pardos e 18,2\% de negros acometidos. No entanto, devido ao pequeno tamanho da amostra, não podemos afirmar que este seja o padrão de distribuição da doença.

A fragilidade óssea é um sinal indicativo da $\mathrm{Ol}^{8}$, sendo que as fraturas muitas vezes podem ser reconhecidas antes mesmo do nascimento ${ }^{8,15,16}$. As fraturas intra-uterinas podem ser detectadas por meio de ultra-sonografia fetal, já no primeiro trimestre da gestação ${ }^{8,15,17}$. Estes pacientes são classificados, segundo os critérios de Sillence et al. ${ }^{4}$, como portadores do tipo II de Ol, sendo esta a forma mais rara e grave 


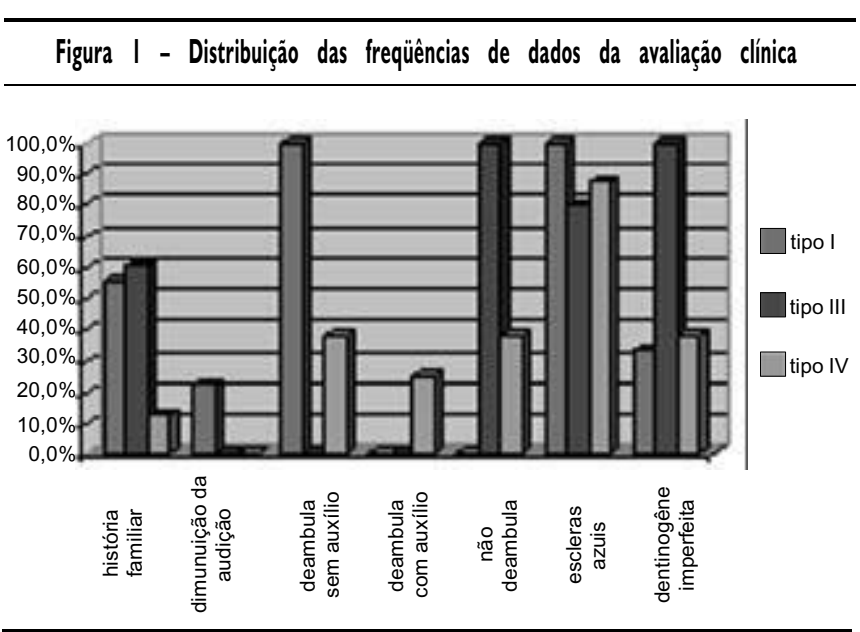

Fonte: Serviço de arquivo médico e estatístico da I rmandade da Santa Casa de Misericórdia de São Paulo

\section{Figura 2 - Número de fraturas em relação ao tipo da doença}

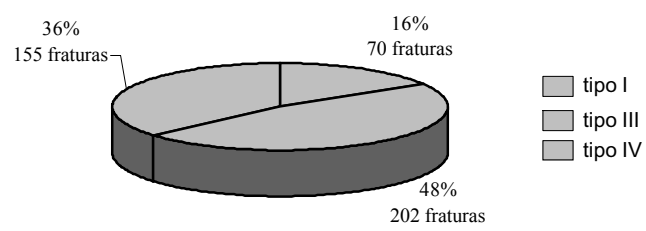

Fonte: Serviço de arquivo médico e estatístico da Irmandade da Santa Casa de Misericórdia de São Paulo

\section{Figura 3 - Distribuição das médias de desvios-padräo quanto à estatura e densidade de massa óssea (DMO) abaixo da normalidade nos diferentes tipos da doença}

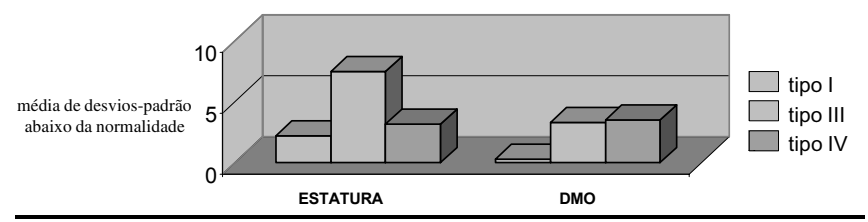

Fonte: Serviço de arquivo médico e estatístico da Santa Casa de Misericórdia de São Paulo

da doença. Caracteristicamente, causam o óbito da criança antes ou logo após o nascimento 5,6,9,18. Isso justifica em parte o fato de não termos na nossa casuística portadores deste tipo de Ol no período de realização deste trabalho.

As fraturas ocorrem nos primórdios da infância, atingem preferencialmente os membros inferiores e tornam-se menos comuns após a puberdade. Sua frequêencia e precocidade de início estão diretamente relacionadas com a gravidade da doença $6,8,17,19$.
Na presente avaliação observamos que o número de fraturas e o início de sua ocorrência foram fatores importantes para a classificação, e conseqüentemente, para estabelecer o prognóstico. Encontramos diferença estatisticamente significante ao compararmos o número de fraturas nos pacientes dos tipos III e IV da doença em relação aos portadores do tipo I, sendo esta última a forma mais branda e de melhor prognóstico quanto ao curso natural da doença. Observamos também que, na média, o primeiro episódio de fratura ocorreu mais precocemente no tipo III, seguido dos tipos IV e I.

As escleras azuladas e a dentinogênese imperfeita são também características marcantes quando presentes nos pacientes portadores de $\mathrm{Ol}^{2}$. A maioria dos nossos pacientes $(90,9 \%)$ apresentava escleras azuis, e caracteristicamente, estava presente em todos os pacientes do tipo I, sendo que este achado coincide com a literatura ${ }^{2,6}$. A dentinogênese imperfeita caracterizada por falha na formação da dentina e maior propensão às cáries é relatada nos portadores de OI do tipo $\mathrm{III}^{2,13}$, e isto foi realmente encontrado em todos os nossos pacientes do tipo III, e em $50 \%$ do total dos pacientes analisados.

A diminuição da acuidade auditiva decorrente da otosclerose é do tipo condutiva, inicia-se na $2^{2}$ ou $3^{a}$ década de vida, e tem caráter gradual e progressivo. Ocorre em até $40 \%$ dos pacientes portadores do tipo I da doença e é pouco freqüente nos demais grupos ${ }^{6,20}$. Tal deficiência foi observada em 22,2\% dos nossos pacientes com o tipo I de Ol, não afetando os pacientes dos demais tipos.

As deformidades esqueléticas são freqüentes, predominam nos membros inferiores e são decorrentes de fraturas viciosamente consolidadas. Estão diretamente relacionadas com a gravidade do acometimento e interferem no prognóstico da marcha $a^{8,13,14,21,22}$. Na avaliação do estado deambulatório dos nossos pacientes constatamos que, caracteristicamente, todos os pacientes do tipo I eram deambuladores, enquanto que os portadores do tipo III nunca tinham deambulado. Os portadores do tipo IV apresentavam características deambulatórias variáveis, mas diferentemente daqueles do tipo III, mesmo os que atualmente não deambulam, fizeram-no em algum momento de suas vidas.

A baixa estatura é outro achado comum nos pacientes portadores de $\mathrm{O}^{10,23,24,25}$. Estaturas abaixo do percentil 2,5 foram observadas em $68,2 \%$ dos nossos pacientes. Esta constatação pode ser útil para a classificação dos portadores de Ol, pois há differença estatisticamente significante na comparação das estaturas entre os pacientes do tipo III, que são mais baixos que os pacientes dos tipos I e IV.

Nos casos mais graves da doença, podem ocorrer deformidades da coluna vertebral, sendo mais comum a escoliose, que ocorre em $20 \%$ a $40 \%$ dos pacientes, e que, além de contribuir para a baixa estatura, é responsável pela diminuição da capacidade cardiopulmonar, limitando assim a expectativa de vida desses doentes ${ }^{25}$. Além da escoliose, pode também ocorrer cifose, impressão basilar e espondilolistese $e^{17,25}$. Encontramos $63,6 \%$ dos pacientes com escoliose, sendo $80 \%$ nos portadores do tipo III da doença, que apresentavam curvas que excediam os 40 graus. Dois destes pacientes, com idades acima de 40 anos, já apresentavam sintomas de insuficiência respiratória.

Faz parte do quadro clínico o aumento da elasticidade com frouxidão cápsulo-ligamentar, tendência aumentada ao sangramento, fáscies 
triangular, alterações da pele e, menos freqüentemente, a hérnia inguinal, os defeitos congênitos tardios e retardo mental ${ }^{5,21}$.

Radiograficamente são encontradas alterações ósseas, que variam em qualidade e quantidade segundo a gravidade da doença. Há em geral uma diminuição do trabeculado ósseo normal, uma diminuição da espessura da cortical e deformidades angulares $8,10,14,20$. Estas deformidades são comuns na convexidade dos ossos longos dos membros inferiores, podendo coexistir com fraturas em várias fases de consolidação num mesmo paciente. Nas formas mais graves existem as chamadas calcificações em "pipoca" na região metafisária dos ossos longos e mais raramente podemos encontrar casos de protrusão acetabular 5,6,10. $^{\text {. }}$

A densidade óssea está diminuída nos doentes com Ol, e isso pode ser investigado por métodos radiográficos, pela densitometria óssea ou pela tomografia da coluna lombar. As radiografias mostram uma diminuição do trabeculado ósseo normal, que pode ser inferida segundo a classificação descrita por Singh et al. ${ }^{26}$. Na análise da avaliação interobservadores sobre o trabeculado ósseo radiográfico e/ou da espessura do osso cortical na região proximal do fêmur, os quatro diferentes pesquisadores concordaram que havia diminuição (osteopenia) em 68,2\% dos nossos pacientes.

A densitometria óssea (DMO) atualmente é um método mais eficaz que a radiografia, pois consegue quantificar objetivamente a diminuição de massa óssea. A DMO também se presta para o seguimento dos pacientes que são tratados clinicamente com bisfosfonados ou outras drogas. Embora Moriwake \& Seino ${ }^{9}$ tenham encontrado a densidade de massa óssea diminuída em todos os seus pacientes com Ol, na nossa casuística isto não foi comprovado. Apenas um paciente ( I I, | \%), entre os nove classificados como do tipo I, apresentou diminuição significante da densidade de massa óssea, segundo o método utilizado. Por outro lado, todos os pacientes portadores dos tipos III e IV apresentaram níveis significantes de diminuição da massa óssea. Existiu diferença estatisticamente significante na comparação dos resultados da DMO dos pacientes do tipo I de OI com aqueles do tipo III e IV (que não apresentavam por sua vez, diferença significante entre si).

Embora a literatura não relate alterações em relação às dosagens do cálcio sérico ${ }^{1,5,9}$, constatamos que $81,8 \%$ dos pacientes avaliados apresentavam hipocalcemia, no entanto, não houve diferença estatística significante entre os subtipos da doença, para podermos utilizar esta variável no diagnóstico diferencial intergrupos.

Apesar de existir uma grande variabilidade de manifestações na OI, a "confirmação do diagnóstico" da doença se faz principalmente pela história clínica e o exame físico. Devem ser investigados os antecedentes familiares, relatos de fraturas com mecanismo de trauma que não se justificariam em pessoas com estrutura músculoesquelética normal; e associação de alterações físicas características como: escleras azuladas, dentinogênese imperfeita, escoliose, baixa estatura e deformidades angulares, principalmente nos membros inferiores. A radiografia, além de propiciar a avaliação das deformidades constatadas no exame clínico, auxilia também no diagnóstico, quando se detecta a diminuição do trabeculado ósseo e o adelgaçamento cortical principalmente no fêmur proximal.
Embora existam sinais sugestivos, é muito importante considerar que não há ainda sinal clínico ou exame complementar patognomônico que confirme o diagnóstico, e assim, deve-se advertir que, principalmente, nos pacientes de menor idade, os casos suspeitos devem ter diferencial com outras doenças ósseas ou mesmo com a síndrome da criança vítima de abuso físico.

\section{CONCLUSÃo}

A classificação destes pacientes, com o objetivo de se determinar seu prognóstico e a melhor forma de tratamento, é de fundamental importância. No entanto, os critérios para classificação propostos por Sillence et al. ${ }^{4}$ nos parecem pouco úteis para a distinção de alguns tipos de apresentação, principalmente entre os pacientes pertencentes aos tipos III e IV. Nestes casos, o fato do paciente nunca ter deambulado e a estatura muito baixa nos parecem as variáveis mais importantes para classificá-los como tipo III, enquanto que para a diferenciação entre os tipos I e IV, a DMO é o exame de maior valia, sendo mais favorável no tipo I.

\section{Agradecimento}

Agradecemos ao Núcleo de Apoio à Publicação da Faculdade de Ciências Médicas da Santa Casa de São Paulo - NAP-SC o suporte técnico-científico à publicação deste manuscrito.

Conflito de interesse: não há.

\section{SUMmary}

ClinICAL, RADIOGRAPHIC AND LABORATORY EVALUATION OF
PATIENTS WITH OSTEOGENESIS IMPERFECTA

BACKGROUND. Osteogenesis imperfecta is a genetic disorder characterized by defects in type / collagen. The main symptom is bone fragility and susceptibility to fractures. Other clinical findings are dentinogenesis imperfecta, blue sclera, early deafness and joint laxity. The purpose of this paper is to establish a practical relationship of the clinical differences between the Sillence's groups.

METHODS. 22 patients were classified according to Sillence et al criteria and submitted tolaboratory tests including blood calcium level and bonedensitometry.

RESULTS. All clinical and laboratory differences were discussed in the text.

Conclusions. Differences suchas results that were found in walking ability, height and bone densitometry were significant and may help to classify patients and to establish prognosis. [Rev Assoc Med Bras 2005; 5 I(4): 214-20]

KEY WORDS: Osteogenesis imperfecta. Dentinogenesis imperfecta. Blue sclera.

\section{REFERÊNCIAS}

I. Weinstein SL, Buckwalter AJ. Tecidos músculo-esqueléticos e sistema músculo-esquelético. In: Buckwalter JA, Weinstein SL, editores. Ortopedia de Turek: princípios e sua aplicação. São Paulo: Manole; 2000.p. 13-68. 
Santill C et al.

2. Cole WG. The Nicholas Andry Award- 1996. The molecular pathology of osteogenesis imperfecta. Clin Orthop 1997;343:235-48.

3. Dalgleish R. The human type I collagen mutation database. Nucleic Acids Res_1997;25: I 81-7.

4. Sillence DO, Senn A, Danks DM. Genetic heterogeneity in osteogenesis imperfecta. J Med Genet 1979; 16:101-16.

5. Engelbert RH, Pruijs HE, Beemer FA, Helders PJ. Osteogenesis imperfecta in childhood: treatment strategies. Arch Phys Med Reabil 1998;79: 1590-4.

6. Minch CM, Kruse RW. Osteogenesis imperfecta: a review of basic science and diagnosis. Orthopedics 1998;21:558-67.

7. Shoenfeld Y. Osteogenesis imperfecta. Review of the literature with presentation of 29 cases. Am J Dis Child 1975; 1 29:679-87.

8. Albright JA, Grunt JA. Studies of patients with osteogenesis imperfecta. J Bone Joint Surg Am 1971;53: 1 4 I 5-25.

9. Moriwake T, Seino Y. Recent progress in diagnosis and treatment of osteogenesis imperfecta. Acta Paediatr Jpn 1997;39:52 I-7.

10. Trelstad RL, Rubin D, Gross J. Osteogenesis imperfecta congenita: evidence for a generalized molecular disorder of collagen. Lab Invest 1977;36:501-8.

I I. Tachdjian MO. Displasia do osso. In: Mihran O, Tachdjian MO, editores. Ortopedia pediátrica. 2a ed. São Paulo: Manole; 1995. p.761-89.

12. Solomons CC, Millar EA. Osteogenesis imperfecta - new perspectives. Clin Orthop 1973;96:299-303.

13. Chines A, Boniface A, McAlister W, Whyte M. Hypercalciuria in osteogenesis imperfecta: a follow-up study to assess renal effects. Bone 1995; 1 6:333-9.

14. Marques RM, Marcondes E, Berquó E, Prani R, Yunes J. Crescimento e desenvolvimento pubertário em crianças e adolescentes brasileiros. II. Altura e peso. São Paulo: Editora Brasileira de Ciências; 1982.

15. Omati AW, Chaves FR. Osteogênese imperfeita intra-útero. Relato de um caso. Rev Imagem 1985;7:29-30.

16. Galera MF, Brunoni D, Patrício FRS, Cernach MCSP, Moron AF, Lederman HM. Osteogênese imperfeita: forma letal. Estudo genético clínico, radiológico e anatomopatológico de três casos. Rev Paul Pediatr |997; | 5: | 87-9|.
17. Jones SJ, Glorieux FH, Travers R, Boyde A. The microscopic structure of bone in normal children and patients with osteogenesis imperfecta: a survey using backscattered electron imaging. Calcif Tissue Int 1999;64:817.

18. Berge LN, Marton V, Tranebjaerg L, Kearney MS, Kiserud T, Oian P. Prenatal diagnosis of osteogenesis imperfecta. Acta Obstet Gynecol Scand 1995; 74:32।-3.

19. Ring D, Jupiter JB, Labropoulos PK, Guggenheim JJ, Stanitsky DF, Spencer DM. Treatment of deformity of the lower limb in adults who have osteogenesis imperfecta. J Bone Joint Surg Am 1996;78:220-5.

20. Astrom E, Soderhal S. Beneficial effect of bisphosphonate during five years of treatment of severe osteogenesis imperfecta. Acta Paediatr 1998;87:64-8.

21. Harrison WJ, Rankin KC. Osteogenesis imperfecta in Zimbabwe: a comparison between treatment with intramedullary rods of fixed-length and self-expanding rods. J R Coll Surg Edinb 1998;43:328-32.

22. Antoniazzi F, Bertoldo F, Mottes M, Valli M, Sirpresi S, Zamboni G, et al. Growth hormone treatment in osteogenesis imperfecta with quantitative defect of type I collagen synthesis. J Pediatr 1996; | 29:432-9.

23. Pradella ML, Araujo AQC. Osteogenesis imperfecta tarda e invaginação basilar. Relato de caso. Rev Bras Neurol 1987;23:65-7.

24. Steiner RD, Pepin M, Byers PH. Studies of collagen synthesis and structure in the differentiation of child abuse from osteogenesis imperfecta. J Pediatr 1996; | 28:542-7.

25. Engelbert RH, Gerver WJ, Breslau-Siderius LJ, Van der GraafY, Pruijs HE, Van Doorne JM, et al. Spinal complications in osteogenesis imperfecta: 47 patients I- 16 years of age. Acta Orthop Scand 1998;69:283-6.

26. Singh $M$, Nagrath AR, Maini PS. Changes in trabecular pattern of the upper end of the femur as an index of osteoporosis. J Bone Joint Surg Am 1970;52:457-67.
Artigo recebido: 12/04/04 Aceito para publicação: I5/I 0/04 